\title{
Dynamiques d'adaptation des femmes aux transformations des systèmes laitiers périurbains en Afrique de I'Ouest
}

\author{
M. Schneider ${ }^{1 *}$ H. Kouyaté ${ }^{2}$ G. Fokou ${ }^{1}$ \\ J. Zinsstag ${ }^{3}$ A. Traoré ${ }^{2}$ M. Amadou ${ }^{1}$ B. Bonfoh ${ }^{4}$
}

\section{Mots-clés}

Produit laitier - Collecte de lait Modernisation - Rôle des femmes Changement social - Afrique de l'Ouest.

\begin{abstract}
Résumé
En Afrique de l'Ouest, les nouveaux modèles de collecte du lait par des unités de transformation sont le reflet des innovations technologiques et institutionnelles promues par les politiques et les projets de développement de la filière. Or, la création des centres de collecte, des minilaiteries ou d'industries laitières bouleverse l'organisation sociale traditionnelle du système laitier et contribue à une déféminisation de la filière. Certaines femmes sont dépossédées de l'activité de collecte - commercialisation et de la gestion des revenus du lait au profit des bergers ou propriétaires hommes. Ce processus est ainsi susceptible de contribuer à la perte de leur autonomie financière. Une étude de cas menée au Mali auprès de trois femmes - transformatrices, exerçant autour de la minilaiterie de Kasséla à 40 kilomètres de Bamako, a permis d'apporter un éclairage à ces évolutions. A partir de questionnaires et d'entretiens semi-structurés, l'étude a permis de mesurer le degré d'adaptation des femmes dans cette dynamique de transformation de la filière laitière locale. Face à l'émergence d'une minilaiterie gérée par les hommes, elles ont initié plusieurs mécanismes de résilience. Cela passait par l'incorporation de la poudre de lait importé, la diversification des produits qu'elles mettaient sur le marché et la fidélisation de la clientèle. Ces stratégies leur ont permis de sécuriser leurs moyens d'existence malgré la prise des parts de marché par les laiteries. Avec des techniques artisanales, chacune d'elles transformaient en moyenne 468 litres de lait par jour, soit le tiers de la production totale de la laiterie de Kasséla, avec un rapport avantage sur coût supérieur à 20 p. 100. Aujourd'hui, les projets de développement laitier tendent de plus en plus à discuter des implications sociales des innovations qu'ils proposent.
\end{abstract}

\section{INTRODUCTION}

De nos jours, on observe dans les sociétés pastorales une diversification croissante des revenus générés à la fois par des activités pastorales et par d'autres types de travaux. Au sein de l'activité pastorale, la diversification des revenus procède d'une marchandisation de plus en plus marquée des systèmes d'élevage, c'est-à-dire d'un recours croissant à la vente des divers « sous-produits » de

1. Institut du Sahel, BP 1530, Bamako, Mali.

2. Institut polytechnique et rural de Katibougou, Bamako, Mali.

3. Swiss Tropical and Public Health Institute, Basel, Switzerland.

4. Centre suisse de recherches scientifiques en Côte d'Ivoire.

* Auteur pour la correspondance

Tél. : +235655715160

E-mail : mariama.schneider@gmail.com l'élevage (viande, beurre, lait, etc.) dont se chargent notamment les femmes (18).

En Afrique de l'Ouest, les femmes sont au cœur de l'économie laitière. Elles participent de manière directe à la production, à la collecte, mais surtout à la transformation et à la commercialisation du lait et des produits laitiers, ainsi qu`à la gestion des revenus générés (1). Historiquement, le secteur laitier fournit aux femmes d'éleveurs un revenu quotidien indispensable à la sécurité du ménage et au bien-être de la famille.

Or, actuellement, les systèmes de production pastoraux sont en pleine mutation : modification des itinéraires de transhumance, semi-intensification, diminution des risques sanitaires, modification des structures familiales, etc. En particulier, on assiste à des changements dans les comportements des éleveurs en matière de commercialisation de leurs produits $(8,18)$. En effet, autour des 
grandes villes se sont développés des bassins laitiers qui ont permis la création des minilaiteries. Avec la présence des laiteries, le système traditionnel de gestion du lait et des revenus du lait détenu par les femmes est en passe d'être totalement bouleversé, les hommes ayant détourné des activités de collecte et de transport du lait vers les nouvelles unités de transformation (9).

Ainsi, les transformations des systèmes de production pastorale affectent les interactions entre les acteurs de la filière laitière aussi bien au niveau de la communauté que du ménage. Il en découle de nombreux changements socio-économiques qui ne contribuent pas toujours au bien-être des couches les plus vulnérables. C'est ainsi qu'en milieu périurbain l'on assiste à une " déféminisation » des activités de gestion du lait et des produits laitiers locaux $(1,7,8,9$, 10, 11, 12, 15). Dans cette dynamique, les femmes d'éleveurs, qui avaient jusqu'ici occupé une place prépondérante dans l'économie paysanne pastorale, sont susceptibles de perdre leur pouvoir économique et pourraient se retrouver dans une situation de dépendance financière quasi exclusive vis-à-vis de leurs époux. Ce changement s'opère à deux niveaux : premièrement au niveau des ménages d'éleveurs où les femmes cèdent leurs droits sur le lait ; deuxièmement dans la collecte, la transformation et la commercialisation du lait, où elles tendent à perdre des parts de marché dans la filière laitière, mettant en péril leurs activités génératrices de revenus ( 7 , $8,9,10)$

Cependant, il apparait clairement qu'autour des villes d'Afrique de l'Ouest (comme par exemple Bamako au Mali), la femme reste encore un acteur clé dans la production, la transformation et la commercialisation du lait. Cette persistance de l'importance économique des femmes semble s'expliquer par la grande variété de produits (lait pasteurisé, ghee, féné) commercialisés en dehors des circuits de collecte et de transformation d'une laiterie ou d'une industrie laitière reconnue (5).

Si ces mutations ont fait l'objet de plusieurs diagnostics au niveau des ménages pastoraux, elles ont été encore assez peu analysées au niveau du commerce et de la transformation. La présente étude a été initiée afin de documenter cette dynamique. Elle s'appuie sur l'étude des stratégies d'adaptation des femmes vis-à-vis des transformations des systèmes de commercialisation du lait en zone périurbaine de Bamako au Mali. Elle a eu pour objectif d'analyser le processus de "déféminisation » des activités de transformation et de commerce du lait, de relever les stratégies d'adaptation des femmes aux changements en cours dans le commerce, et d'évaluer les conséquences socio-économiques et techniques de cette adaptation, à partir d'une étude de cas d'un petit groupe de femmes - transformatrices. Elle visait à apporter un éclairage sur la dynamique plus globale des systèmes laitiers autour des grandes villes d'Afrique de l'Ouest.

\section{MATERIEL ET METHODES}

\section{Zone d'étude}

L'étude s'est déroulée entre juin et novembre 2006 à Kasséla, un village de la commune de Baguinéda Camp situé à 42 kilomètres au nord-est de Bamako. Estimée à 1435 habitants, la population de Kasséla est composée de plusieurs ethnies, avec les effectifs les plus importantes chez les Bambara et les Peuls (3). Les principales activités sont l'élevage, l'agriculture et le petit commerce (produits manufacturés, bois d'œuvre et de chauffe, charbon). Le village de Kasséla est bordé par la forêt classée de la Faya, exploitée par les villageois pour leur subsistance. La présence de cette forêt fait de Kasséla un espace pastoral de grande importance. C'est une zone reconnue pour son cheptel bovin et son potentiel laitier important dont l'excédent permet d'approvisionner les populations de la capitale. Le mode d'élevage pratiqué est semi-extensif avec une forte tendance à la sédentarisation. Les éleveurs ont recourt à la complémentation alimentaire mais pratiquent une transhumance d'amplitude variable.

Depuis 1998, un centre de collecte, de transformation et de commercialisation du lait a été implanté à Kasséla. Le lait provient de fermes issues des villages de Mofa, Diko, Karakan, Soro, Kocoum, Gomougouko et Yayabougou qui sont situés dans un rayon de 15 kilomètres autour de Kasséla. Les producteurs qui livrent du lait au centre de collecte sont organisés en coopérative. Une partie des produits de cette minilaiterie sont vendus sur place et le reste est acheminé vers la ville de Bamako (3).

\section{Echantillonnage}

Cette étude s'est appuyée principalement sur trois femmes impliquées dans le commerce du lait. Dans le village de Kasséla, ces trois femmes étaient les seules personnes qui, en dehors de la minilaiterie, exerçaient une activité de collecte et de transformation du lait de manière permanente. Il s'agissait d'analyser les changements qui affectaient ce groupe de femmes - transformatrices dans leur activité et de déterminer comment elles s'adaptaient à ces transformations. Cependant pour mieux comprendre la dynamique qui s'opère autour des femmes, l'étude s'est en outre intéressée à un certain nombre d'acteurs. Cet échantillon comprenait :

- le gérant du centre de collecte et certains membres de la coopérative laitière ;

- des collecteurs $(n=23)$ de lait qui approvisionnaient les transformatrices ; des fiches de suivi des volumes de lait collectés chez les 23 collecteurs ont été dressées ;

- des clients grossistes $(n=60)$; ces commerçants ont été interviewés pour connaître leurs circuits d'approvisionnement et leurs interactions avec les femmes ;

- des clients consommateurs de Bamako ( $\mathrm{n}=90)$; ces consommateurs choisis au hasard dans les différents quartiers de Bamako ont été interviewés sur la perception de la qualité du lait et des produits laitiers vendus par ces femmes et sur les questions de fidélisation de la clientèle.

Le recours à ce type d'informateurs a eu pour objectif de comprendre les relations et les logiques de remodelage des interactions entre les acteurs du système de production laitière depuis l'implantation de la laiterie. La compréhension de ces interactions a permis de saisir les dynamiques complexes autour de la filière laitière dans cette région. Le travail s'est par ailleurs appuyé sur plusieurs études et recherches menées récemment sur la laiterie de Kasséla et sur les produits laitiers fabriqués par les transformatrices $(15,17,19)$.

\section{Collecte des données}

Au cours de cette étude, des données sociologiques, économiques et portant sur la qualité physico-chimique des produits ont été collectées. Dans un premier temps, des entretiens semi-structurés ont été réalisés avec les membres de la coopérative laitière de Kasséla, les transformatrices et les collecteurs de lait. Ces entretiens ont permis de collecter des données qualitatives sur les transformations intervenues dans la filière laitière, la reconstitution de l'historique de leur activité et des relations avec les autres acteurs, ainsi que les stratégies d'adaptation des femmes. Un questionnaire séparé a été administré au gérant de la laiterie de Kasséla pour comprendre la nature des relations de la laiterie avec les femmes.

Pour mesurer les incidences de ces dynamiques d'adaptation des femmes face aux transformations des systèmes laitiers, le suivi de 
la collecte, de la transformation et de la commercialisation du lait a été effectué durant le mois de juin (fin de saison sèche) et le mois de juillet (début de saison des pluies) à l'aide de fiches. Le suivi des dépenses et des recettes chez les trois femmes a également été établi. Les fiches ont permis de recueillir des données économiques (prix des équipements, des intrants, les services et les fluctuations des prix du lait) afin de déterminer les coûts engendrés par l'activité des femmes, leurs revenus, le bénéfice et la rentabilité de l'activité, et d'en faire une analyse financière.

Des données sur la qualité hygiénique du lait collecté par les femmes ont été recueillies. Au niveau de la minilaiterie de Kasséla, un contrôle de qualité a été effectué sur tous les laits « réceptionnés au quai ». Ce contrôle de qualité a été réalisé grâce aux tests d'acidité et de densité, tests reconnus par la minilaiterie comme étant les principaux indicateurs de la qualité du lait. Ces deux tests ont porté sur cinq échantillons de lait cru prélevés au hasard chez chacune des trois femmes et testés successivement à l'alcool à $70^{\circ}$ (test d'acidité) puis au thermo-lactodensimètre (test de la densité/test de mouillage). D'autres facteurs doivent être pris en considération pour déterminer la qualité hygiénique du lait traité par les femmes (mode de transformation du lait, conditionnement et transport du lait). Cependant, l'objectif poursuivi à travers ces tests de contrôle de qualité était de comparer le lait passant par ce que certains chercheurs appellent le « circuit souillé » (femmes) avec celui du « circuit sain » (laiterie) (15) pour mesurer les caractéristiques biochimiques du lait proposé par les femmes. L'objectif de ces analyses était ainsi de comprendre les ressorts « techniques » de l'activité des transformatrices et de comparer leurs produits à ceux issus du centre de collecte de Kasséla.

\section{Validation des résultats}

Les résultats de l'étude ont fait l'objet d'une restitution au cours d'une rencontre avec les femmes à Kasséla. Cette étape a permis, d'une part, de recueillir les commentaires et observations de ces femmes sur les conclusions et les recommandations de l'étude, et, d'autre part, d'approfondir l'analyse de l'activité des femmes, de leurs performances financières et d'évoquer les options futures.

\section{RESULTATS}

\section{Acteurs de la filière laitière artisanale dans la zone périurbaine de Bamako}

Plusieurs acteurs et groupes d'acteurs participent à la production, à la transformation et à la commercialisation du lait dans la périphérie urbaine de Bamako. Cette étude s'est principalement intéressée à trois catégories d'acteurs dont les interactions permettaient de cerner en particulier la dynamique des activités féminines dans la filière laitière : les transformatrices, leurs fournisseurs, et la laiterie qui est apparue parfois comme concurrente, parfois comme partenaire.

\section{Caractéristiques et organisation des tâches des femmes laitières}

Les trois femmes suivies dans le cadre de cette étude exerçaient les activités de collecte, de transformation et de commercialisation du lait depuis plusieurs années : 15 ans pour la première (F1), 6 ans pour la deuxième (F2) et 22 ans pour la troisième (F3). Elles n'avaient pas reçu de formation de base et avaient démarré la collecte de lait sur fonds propres. Elles ne disposaient pas d'agrément et n'étaient pas officiellement reconnues. De plus, elles ne payaient pas de taxes et leur lait n'était pas contrôlé sur le plan hygiénique. Par ailleurs, elles n'étaient organisées ni en groupement, ni en association ou coopérative. Pourtant, malgré le caractère informel de leur activité, ces femmes avaient mis en place de véritables entreprises. Elles jugeaient leur activité rentable car elle leur permettait de couvrir certains besoins de la famille.

Ces femmes étaient âgées de 31 (F2), 49 (F1) et 62 ans (F3). Elles étaient toutes les trois mariées et avaient chacune à charge cinq personnes en moyenne. L'une d'entre elles était peule (F1) tandis que les deux autres étaient sarakolé. Pour elles, le commerce du lait constituait un moyen de générer des moyens d'existence substantiels plutôt qu'un moyen d'expression d'une culture pastorale. Leur personnel était le plus souvent constitué des membres de la famille, en général des filles dont la principale tâche était la vaisselle, la transformation et le conditionnement du lait. La contribution des membres de la famille en termes de main d'œuvre a été estimée à 60 p. 100 des tâches (F1), 56 p. 100 (pour F2) et 50 p. 100 (pour F3) (tableau I). Ces femmes contribuaient donc à la formation des membres de la famille et à l'emploi des jeunes filles au même titre que la laiterie.

\section{Laiterie de Kasséla}

La laiterie a été mise en place en 1998 avec l'appui du Projet de développement de l'agriculture périurbaine de Bamako (Pdap). Il s'agissait de créer un centre de collecte ayant pour fonction d'apporter des services aux producteurs et de mobiliser les excédents de production pour les industries installées à Bamako. Ce centre était géré par un comité mis en place par la coopérative d'éleveurs et s'appuyait sur le principe « lait contre services ». Les producteurs étaient encouragés à fournir du lait à la coopérative qui devait en retour leur faciliter l'accès à l'aliment pour bétail et aux soins vétérinaires. La collecte était artisanale avec des techniques de contrôle régulier (test à l'alcool et densité). Les femmes ont manifesté très peu d'intérêt pour la coopérative, ce qui était compréhensible compte tenu du fait que le revenu du lait dans les ménages était de plus en plus détenu par les hommes. Au moment de l'étude, une seule femme continuait à faire partie de la coopérative. Jusqu'en 2003, le volume moyen de collecte était estimé à 210 L/jour.

En 2004, au travers de l'appui du projet Lait sain pour le Sahel, le centre de collecte a évolué de manière importante grâce à l'appui technique et à la facilitation de l'accès à des services techniques et financiers. Il a été transformé en une minilaiterie employant dix personnes à plein temps. Au moment de l'étude, la laiterie était approvisionnée en moyenne par 42 collecteurs et traitait près de $1500 \mathrm{~L} /$ jour. Dès sa réception, la minilaiterie soumettait le lait aux tests de contrôle de qualité suivants pouvant entraîner soit son acceptation soit son rejet :

- test de non-conformité des récipients, le lait arrivant au quai de réception dans des récipients sales était rejeté ;

- test de densité (mouillage) avec un lactodensimètre qui consistait à vérifier si le producteur n'avait pas ajouté de l'eau dans le lait ;

- test d'acidité qui permettait de vérifier si le lait n'était pas déjà acide (coagulation).

$\mathrm{Au}$ début (2004), la minilaiterie rejetait en moyenne 10 p. 100 du lait qu'elle recevait pour des raisons de mouillage, d'acidité ou de

\section{Tableau I}

Répartition du nombre d'employés par femme à Kasséla

\begin{tabular}{|lcccc}
\hline Femme & $\begin{array}{c}\text { Employé à } \\
\text { temps plein }\end{array}$ & $\begin{array}{c}\text { Employé à } \\
\text { temps partiel }\end{array}$ & $\begin{array}{c}\text { Membre } \\
\text { famille }\end{array}$ & Total \\
\hline F1 & 3 & 2 & $3(60 \%)$ & 5 \\
F2 & 6 & 3 & $5(56 \%)$ & 9 \\
F3 & 5 & 3 & $4(50 \%)$ & 8 \\
\hline
\end{tabular}


non-conformité des récipients. En 2006, au moment de l'étude, la qualité du lait livré par les collecteurs s'était nettement améliorée et le taux de rejet du lait avait nettement baissé (3 p. 100 en 2006).

La laiterie avait également réussi à réduire les pertes en lait au cours du traitement. Ces pertes étaient passées de 6 p. 100 en 2004 à 3 p. 100 en 2005 puis à 1,5 p. 100 en 2006. Elles étaient essentiellement liées à des défauts de fermentation (dus aux résidus d'antibiotiques) et à la dénaturation du lait lors du chauffage (lait gardé longtemps avant pasteurisation).

Le déficit de savoir-faire en marketing, et l'absence d'une source d'énergie électrique et d'une chaîne de froid étaient les deux principaux handicaps de la coopérative. La laiterie commercialisait deux produits : le lait frais pasteurisé et le féné (lait traditionnel spontanément fermenté). Elle achetait le litre de lait frais réceptionné au quai au prix « harmonisé » de $250 \mathrm{Fcfa}$. Après chauffage à $80^{\circ} \mathrm{C}$ pendant plusieurs minutes, le lait était refroidi puis revendu en gros et au détail au prix de $300 \mathrm{Fcfa} / \mathrm{L}$. Le féné était vendu à $350 \mathrm{Fcfa} / \mathrm{L}$. La marge que la laiterie obtenait par litre de lait était de l'ordre de 34 Fcfa en 2006. Le rapport avantage ou bénéfice sur coûts était évalué à 6,5 p. 100 (tableau II).

\section{Fournisseurs et volume de lait collecté par les femmes}

Le lait traité par les femmes était fourni par 23 collecteurs différents (ou fournisseurs) : 2 pour F1, 11 pour F2 et 10 pour F3. Il s'agissait de bergers âgés de 17 à 60 ans provenant de huit villages répartis dans un rayon de 15 kilomètres autour de Kasséla. Le moyen de transport des deux tiers des collecteurs était le vélo (69 p. 100 d'entre eux). Parmi ces collecteurs, les bergers peuls

\section{Tableau II}

Résultat annuel de la laiterie de Kasséla en 2006

\begin{tabular}{|c|c|c|c|}
\hline Charges & $\begin{array}{c}\text { Valeurs } \\
\text { (Fcfa) }\end{array}$ & Recettes & $\begin{array}{c}\text { Valeurs } \\
\text { (Fcfa) }\end{array}$ \\
\hline Achat lait & 91631581 & Vente de lait & 109949296 \\
\hline Personnel & 2002500 & - & - \\
\hline Autres charges & 9647960 & - & - \\
\hline Total & 103282041 & Total & 109949296 \\
\hline $\begin{array}{l}\text { Marges } \\
\text { bénéficiaires } \\
\text { brutes }\end{array}$ & 6667255 & $\begin{array}{l}\text { Marges } \\
\text { bénéficiaires } \\
\text { brutes }\end{array}$ & 6667255 \\
\hline Avantages/coûts & $6,5 \%$ & Avantages/coûts & $6,5 \%$ \\
\hline
\end{tabular}

Source : rapport VSF Suisse, Bamako, 2007 étaient majoritaires (69 p. 100), puis venaient les bergers bambara (25 p. 100) et sarakolé (4 p. 100). A Kasséla, 60 p. 100 des collecteurs de lait pratiquaient cette activité de façon saisonnière alors que 40 p. 100 en faisaient une activité principale toute l'année. Il s'agissait en majorité de bergers rémunérés mensuellement par les propriétaires des animaux dont ils avaient la garde, et qui commercialisaient une partie du lait issu de ce cheptel. Les femmes entretenaient de très bonnes relations sociales et économiques avec les collecteurs. En raison de ces relations personnelles très fortes, il a été difficile de connaître avec précision les volumes vendus par chacun des bergers et de s'informer sur les éventuelles tricheries (le mouillage du lait par exemple).

Avec deux collecteurs seulement, F1 recevait en moyenne 84 litres de lait par jour. En revanche, elle commercialisait plus de $150 \mathrm{~L} /$ jour en moyenne, du fait du mélange du lait de vache avec du lait reconstitué à partir de la poudre de lait. En effet, 44 p. 100 du lait transformé par F1 était du lait reconstitué incorporé dans le lait frais (qualifié parfois de lait «pur») qu'elle avait reçu des collecteurs (tableau III). F2 incorporait aussi du lait reconstitué au moment de l'enquête mais de manière très marginale (moins de 0,5 p. 100 de la quantité totale de lait). Elle transformait en moyenne $640 \mathrm{~L} /$ jour. La quantité journalière de lait transformé par F3 était de $680 \mathrm{~L}$; parmi les trois femmes, elle était la seule qui n'utilisait pas du tout de lait en poudre.

Les quantités de lait transformées par ces trois femmes ont été importantes mais elles ont fluctué suivant les saisons. En fin de saison sèche (c'est-à-dire au début du mois de juin), le nombre de collecteurs ainsi que le volume de lait collecté par les femmes ont considérablement diminué en raison du départ de certains bergers vers leurs zones de transhumance et de la faible disponibilité en ressources alimentaires pâturées. En revanche, pour la période de juillet, les volumes ont augmenté. En moyenne sur les deux mois, les trois femmes ont transformé 1472 litres de lait par jour, c'està-dire presque le même volume de lait que la laiterie (1 $500 \mathrm{~L} /$ jour) (tableau III). Ces grandes quantités de lait collectées par les femmes au moment où de nombreux producteurs commercialisaient leur lait à la laiterie se justifiaient par le fait que leur approvisionnement se faisait par l'intermédiaire d'un circuit qui échappait au contrôle des producteurs ou des collecteurs de la laiterie : il s'agissait pour l'essentiel des quantités de lait destinées à la consommation des bergers ou soustraites par ces derniers aux animaux dans les élevages dont ils avaient la charge.

\section{Particularités et stratégies des femmes}

\section{Stratégies de transformation du lait cru}

Les femmes de Kasséla avaient adopté chacune des techniques de transformation du lait cru différentes qui leur permettaient de satisfaire leur clientèle. Le procédé de transformation développé par

\section{Tableau III}

Volume moyen de lait traité par jour par les trois femmes en juin et juillet 2006

\begin{tabular}{lcccccc} 
Femme & $\begin{array}{c}\text { Nb. de } \\
\text { fournisseurs }\end{array}$ & $\begin{array}{c}\text { Moyenne/ } \\
\text { fournisseur } \\
\text { (L/jour) }\end{array}$ & $\begin{array}{c}\text { Vol. moyen de } \\
\text { lait pur collecté } \\
\text { (L/jour) }\end{array}$ & $\begin{array}{c}\text { Vol. moyen de } \\
\text { lait vendu } \\
\text { (L/jour/femme) }\end{array}$ & $\begin{array}{c}\text { Vol. moyen de } \\
\text { lait reconstitué } \\
\text { incorporé (L/jour) }\end{array}$ & $\begin{array}{c}\text { Vol. moyen de } \\
\text { lait reconstitué } \\
\text { incorporé (\%) }\end{array}$ \\
\hline F1 & 2 & 42 & 84 & 150 & 66 & 44 \\
F2 & 11 & 58 & 638 & 640 & 2 & 0,31 \\
F3 & 10 & 68 & 680 & 680 & 0 & 0 \\
Total & 23 & 64 & 1472 & 1472 & 68 & 4,62
\end{tabular}


F3 lui permettait par exemple de répondre à l'attente de sa clientèle fidélisée en lui fournissant des produits recherchés (ghee) et de qualité organoleptique optimale pour le consommateur malien (féné très épais et crémeux). Par ailleurs, l'utilisation du lait en poudre était une stratégie qui permettait à ces femmes de maintenir le volume de lait traité et donc leur revenu dans les périodes de baisse de production laitière.

Le lait cru entier provenant des collecteurs était chauffé plusieurs minutes à une température comprise entre 80 et $100{ }^{\circ} \mathrm{C}$ dans des marmites, sur des foyers dits « améliorés » utilisant du bois comme combustible. Le film de matière grasse, formé à la surface de la marmite après le chauffage du lait, était récupéré. Le lait chauffé obtenu était donc un lait partiellement écrémé. Celui-ci constituait le premier produit commercialisé chaque jour sous l'appellation de « lait frais ». Il était vendu au prix de $350 \mathrm{Fcfa} / \mathrm{L}$. La matière grasse récupérée chaque jour lors du chauffage du lait était stockée et, au bout d'une semaine, chauffée pour donner une huile que les femmes conditionnaient dans des bouteilles en verre. Ce produit, le ghee, était le second produit commercialisé par les femmes de Kasséla. Il était utilisé essentiellement comme condiment pour les plats en sauce et vendu à $2500 \mathrm{Fcfa} / \mathrm{L}$.

Le féné était le lait fermenté obtenu le lendemain, suite au refroidissement et à la fermentation spontanée du lait chauffé la veille. Il s'agissait donc d'un lait fermenté partiellement écrémé. Mais plusieurs autres techniques étaient utilisées pour fabriquer du féné (figure 1). La plupart du temps, le lait était bouilli jusqu'à l'obtention d'une odeur de brûlé. Ce lait " brûlé » était par la suite cuit à feu doux pendant environ six heures sur un foyer utilisant

\section{Procédéde fabrication du féné et du ghee}

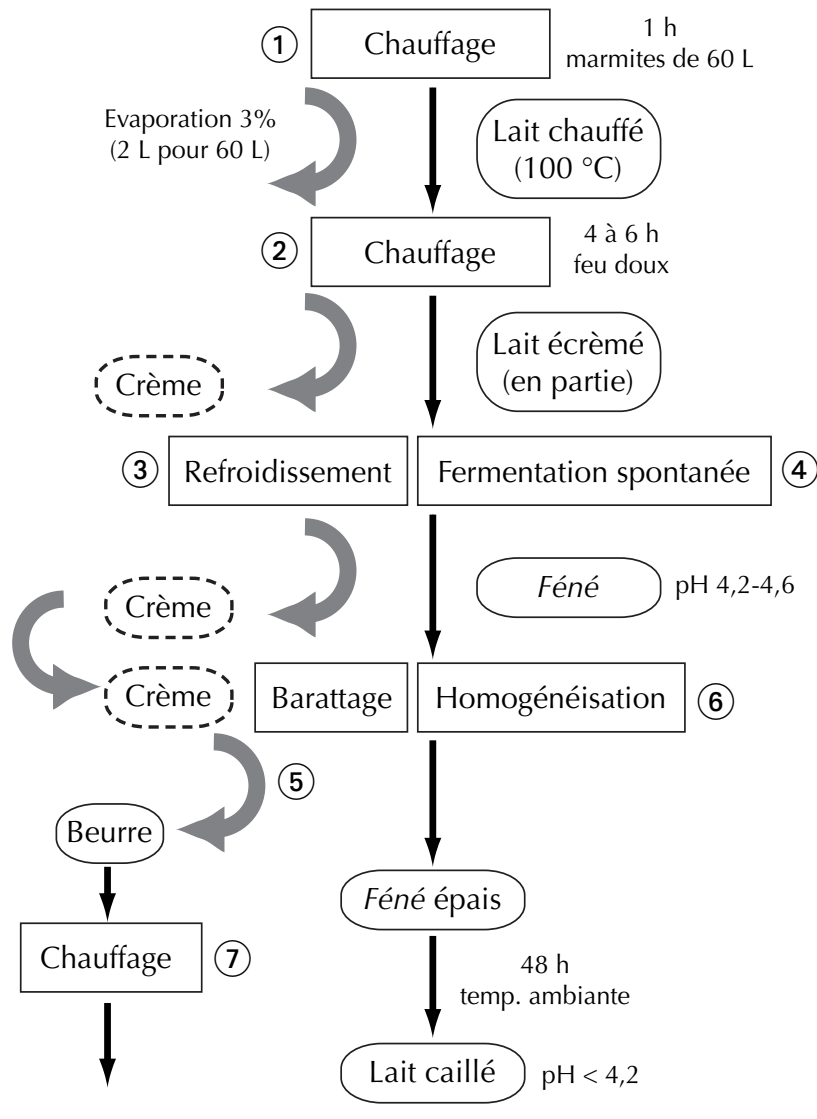

Figure 1 : diagramme de transformation du lait par les femmes - transformatrices. du charbon de bois comme combustible. C'était ce chauffage long et lent qui permettait d'obtenir du féné très épais apprécié par les clients. Il s'agissait alors d'un lait fermenté concentré. Une autre technique de fabrication du féné consistait à ensemencer le lait chauffé avec du yaourt ou du lait fermenté de la veille, pour accélérer la fermentation. Généralement, une nuit suffisait pour la fermentation et, le lendemain matin, apparaissaient trois couches qui s'étaient séparées par gravitation dans la marmite : la couche superficielle constituée de matière grasse avait une consistance épaisse et légère ; la couche intermédiaire (fluide) était constituée du petit-lait ; tandis que le « lait caillé » proprement dit se retrouvait au fond du récipient. La couche superficielle pouvait être isolée pour en extraire le ghee par chauffage. Le reste de la marmite pouvait alors être mélangé puis baratté pendant 10 à 15 minutes afin d'en extraire des globules de beurre puis, par chauffage, du ghee. Le mélange du lait caillé et du petit lait dont une partie de l'eau avait été évaporée constituait ce que les femmes appelaient le féné. Ainsi, le féné correspondait à un lait caillé en partie écrémé et concentré. Il était peu acide (pH 4,2 à 4,6). Le reste du féné non vendu était laissé à reposer et donnait un lait fermenté concentré plus acide $(\mathrm{pH}<4,2)$.

L'utilisation du lait en poudre a été observée chez deux transformatrices sur les trois. La matière première restait le lait cru, mais, en plus, les femmes F1 et F2 incorporaient du lait reconstitué à partir du lait en poudre en proportion importante. Dans le cas de F1, le lait reconstitué constituait 44 p. 100 du lait total, tandis que dans le cas de F2, le lait reconstitué représentait une proportion négligeable du total $(0,31$ p. 100$)$. La transformatrice F3 était la seule qui transformait le lait local pur de vache sans incorporer de lait reconstitué. Les femmes F1 et F2 étaient bien conscientes que cette pratique dénaturait la qualité organoleptique du lait transformé ; mais elles estimaient que cette activité était une option technique leur permettant d'améliorer leurs revenus et les conditions de vie de leurs familles.

\section{Pertes en matière grasse et en eau et stratégies de compensation}

Les techniques de chauffage et de séparation de la crème par gravité pratiquées par les femmes entraînaient des prélèvements importants de matière sur le lait : récupération de la matière grasse et évaporation de l'eau. La production de beurre était de l'ordre de 0,5 litre par jour pour une transformation moyenne de 64 litres par femme par jour. Les pertes par évaporation ont été estimées par les femmes à 3 p. 100 (soit 2 litres sur 60).

Les collecteurs semblaient « compenser » ces pertes en remplissant souvent les bidons au-dessus des niveaux de mesure. Par exemple, pour l'achat de 60 litres de lait, les transformatrices recevaient en général un surplus de deux litres de la part de leurs fournisseurs de lait cru. Chez d'autres collecteurs, les demi-litres n'étaient ni payés, ni comptés et la majorité des collecteurs cédait souvent les demi-litres gratuitement aux femmes pour maintenir leur clientèle en période d'abondance de l'offre.

Ce lait, fourni par les collecteurs, ne subissait pas de contrôle et pouvait parfois être de qualité douteuse : lait mouillé ou acidifié par manque d'hygiène. Par ailleurs, le lait refusé par la laiterie de Kasséla (suite aux tests réalisés au quai de réception) était très souvent acheté par les femmes pour lesquelles les exigences de qualité n'étaient pas les mêmes. Ce lait était par la suite chauffé et transformé selon les procédés décrits plus hauts. Avant le chauffage, les femmes ajoutaient du natron en début d'acidification ou de coagulation, probablement pour réguler le $\mathrm{pH}$. En Afrique de l'Ouest, cette substance est généralement utilisée dans la cuisine dans le but de neutraliser ou réduire l'acidité des légumes (feuilles d'oseille de guinée ou tomates, par exemple). Cette pratique traditionnelle avait été étendue au lait par ces femmes. 
Les transformatrices ajoutaient systématiquement de la potasse au moment du chauffage du lait. Cette pratique était destinée à empêcher la coagulation du lait acide. L'ajout de potasse donnait par ailleurs au lait une couleur jaune-brunâtre ainsi qu'un goût inhabituel. Par ailleurs, le long chauffage du lait lui donnait un goût de brûlé et contribuait à accentuer la couleur jaune-brunâtre du lait après cuisson. Cependant, ces différences de goût et de couleur n'étaient perceptibles que lorsque l'on comparait le lait traité par les femmes avec le lait traité à la laiterie de Kasséla.

\section{Produits mis sur le marché et stratégies de commercialisation}

\section{Gamme de produits et stratégies de vente}

Les femmes présentaient une gamme variée de produits : le lait frais bouilli, le féné, le lait caillé et le ghee. Ainsi, comparativement à la minilaiterie qui ne fournissait que du lait frais et du féné, les femmes enquêtées proposaient des produits plus diversifiés. La transformatrice F3 commercialisait ses produits au détail (bol et louche), tandis que les transformatrices F1 et F2 vendaient leurs produits en gros ou en demi-gros. Toutes les trois vendaient du lait chauffé entier ou partiellement écrémé conditionné dans des bidons en plastique de 10, 20 et 25 litres, et dans des sachets en plastique d'un litre ou d'un demi-litre. Le lait était vendu au niveau des différents marchés de Bamako où ces femmes possédaient un lieu fixe et étaient reconnues comme vendeuses du lait de Kasséla. Elles vendaient le lait au même prix que la laiterie (350 Fcfa le litre de lait chauffé).

En plus du lait chauffé, elles proposaient du lait cru (F1, F2 et F3), du ghee (F2 et F3) et du féné (F3). Si les femmes F1 et F2 ne vendaient pas de féné, cela s'expliquait probablement par le fait qu'elles utilisaient du lait reconstitué, ce qui ne permettait pas d'obtenir un féné de qualité suffisante. La détaillante (F3) obtenait des bénéfices plus importants que les deux autres transformatrices grâce à la fabrication du ghee et à la maîtrise de la technique particulière de fabrication du féné par chauffage long et lent. Par ailleurs, ses produits étaient reconnus dans la localité comme étant «sains», son expérience et son savoir-faire étaient appréciés et sa clientèle locale était plus importante. De plus, la vente au détail (minidose) lui permettait de commercialiser le litre de lait à 500 Fcfa contre 350 Fcfa pour les ventes au litre ou en gros.

\section{Transport et conservation du lait}

L'acheminement du lait vers Bamako s'effectuait dans des minibus. Bien qu'elles aient exercé cette activité depuis plusieurs années, les vendeuses de lait rencontraient des difficultés pour le transport du lait de Kasséla à Bamako, et surtout pour le transport retour des bidons vides. Les chauffeurs des minibus refusaient souvent de transporter leurs bidons de lait à cause des mauvaises odeurs qu'ils dégageaient et de la perte de temps due au chargement et au déchargement. Elles avaient cependant réussi à conclure un accord avec un transporteur du village de Kasséla pour se rendre chaque jour à Bamako. Mais lorsque celui-ci n'était pas disponible, il leur était très difficile de négocier l'appui d'un autre transporteur. Ces femmes réfléchissaient d'ailleurs à la mise en place d'une association entre elles pour régler ce problème.

Les transformatrices de Kasséla ne possédaient pas d'équipements frigorifiques (glacières, réfrigérateurs, etc.) pour la conservation du lait. Elles rencontraient donc d'importantes difficultés dans la conservation du lait non vendu à Bamako. En effet, en cas de mévente, elles ne pouvaient envisager de le retourner à Kasséla, compte tenu des difficultés de transport et de conservation. Elles n'avaient alors pas d'autre choix que de le placer chez des revendeurs qui le prenaient à crédit. Le reste du lait était conservé dans des seaux non couverts au marché et laissé à fermenter. Les risques sanitaires étaient élevés et les pertes importantes. Lors de la première nuit, ce lait se transformait en féné et possédait une valeur marchande certaine ; mais les jours suivants, il perdait de sa valeur en évoluant en lait caillé très acide et moins apprécié des consommateurs.

Ces conditions difficiles de transport et de conservation du lait constituaient des risques de pertes économiques importants et une menace pour la santé des consommateurs (6). Par ailleurs, les manipulations qui s'ajoutaient à l'incorporation incontrôlée du lait en poudre étaient susceptibles d'affecter négativement la qualité sanitaire du lait et des produits laitiers commercialisés par les transformatrices. Malgré tout, les produits des femmes restaient compétitifs.

\section{Facteurs de compétitivité}

\section{Qualité du lait}

Les femmes vérifiaient la qualité du lait par un contrôle organoleptique. Il s'agissait pour elle d'apprécier la qualité du lait à partir de sa couleur, de son aspect, de son odeur et parfois de son goût. Cette vérification permettait de savoir si le lait était en mesure de subir la transformation (après chauffage). Il s'agissait donc d'apprécier sa qualité d'usage. Mais les tests conventionnels d'appréciation et de contrôle de qualité (densité, acidité) n'étaient pas réalisés par les femmes.

Les tests effectués à la laiterie sur cinq échantillons de lait prélevés au hasard chez les femmes ont donné les résultats suivants : 40 p. 100 du lait cru était mouillé, impliquant que certains collecteurs ajoutaient de l'eau dans le lait avant de le livrer aux femmes ; 50 p. 100 des échantillons ont réagi au test à l'alcool, ce qui signifiait que la moitié du lait qui arrivait chez les femmes était déjà partiellement fermenté. Ces résultats ont montré que, comparativement à la laiterie où tout lait testé positif était rejeté, le lait livré aux femmes par les collecteurs était parfois de qualité douteuse. D'ailleurs, les consommateurs interrogés ont affirmé pouvoir clairement faire la différence entre le lait de la laiterie et celui proposé par les femmes.

\section{Prix du lait}

Avant la mise en place de la laiterie (1998), les transformatrices achetaient le litre de lait cru entre 100 et 125 Fcfa par litre chez les collecteurs pour le chauffer ou le transformer en féné. Le litre de lait pasteurisé était ensuite vendu à Bamako au prix de 200 Fcfa. Avec la création de la laiterie, le prix du litre acheté par la laiterie aux collecteurs est monté à 150 Fcfa. En effet, comme la laiterie souhaitait augmenter ses quantités collectées, il lui fallait offrir aux collecteurs un prix plus attractif que celui offert par les transformatrices. Celles-ci avaient d'ailleurs refusé d'adhérer à la coopérative laitière qui gérait la minilaiterie de Kasséla. Selon les entretiens menés auprès des différents acteurs, l'augmentation du prix d'achat du lait par la laiterie (de 125 à $150 \mathrm{Fcfa}$ ) amena les femmes à proposer aux collecteurs un prix encore plus élevé (175 Fcfa). Par la suite, la laiterie décida d'augmenter le prix d'achat du litre à 200 Fcfa, ce qui amena les transformatrices à l'acheter au prix de 225 Fcfa par litre. Finalement, le prix d'achat du litre de lait cru se stabilisa à $250 \mathrm{Fcfa}$. Ainsi, le prix du lait n'a cessé de grimper du fait de la concurrence entre les femmes et la laiterie.

En 2006, le litre de lait pasteurisé (issu de la minilaiterie) était vendu à Bamako entre 300 et 350 Fcfa pour le prix de gros, et de 400 à 600 Fcfa par litre pour le prix de détail, selon la saison et le type de client. Les femmes de Kasséla vendaient le lait chauffé au même prix que la minilaiterie. 


\section{Femmes et laiterie : de la concurrence à la complémentarité}

La «bataille » autour de la fixation des prix au collecteur a montré que pour les femmes la laiterie constituait un concurrent dans la collecte de lait. La responsabilité de la laiterie dans cette hausse des prix est ressortie clairement dans le discours du gérant de la laiterie : «Nous sommes en partie responsables de leurs difficultés car ces femmes exerçaient leur activité depuis longtemps. A l'arrivée de la laiterie, nous avons voulu travailler avec elles. Au départ, il était prévu que nous achetions tous le litre de lait cru à 150 Fcfa chez les collecteurs ou directement chez les producteurs. Or, les transformatrices n'ont pas voulu travailler avec la laiterie. Outre le fait qu'elles redoutaient la concurrence sur l'achat du lait, ces femmes utilisaient pour certaines du lait en poudre, alors que la laiterie avait choisi de ne pas travailler à partir de poudre. Dès lors, une collaboration devenait impossible ».

Au lancement de la laiterie, pourtant, la laiterie apporta un appui aux femmes en leur fournissant du lait cru au prix de gros (200 Fcfa). Mais en 2006, la laiterie approvisionnait ces femmes au même prix que les détaillants (300 Fcfa). Le gérant de la laiterie précisa : "A la laiterie, nos objectifs sont différents de ceux des femmes. Nous, nous voulons que les producteurs puissent tirer profit de leur élevage et qu'un lait de bonne qualité puisse être mis à la disposition des consommateurs (lait pur local). L'objectif des transformatrices vise avant tout la rentabilité économique de leur entreprise. Ces femmes croient que la laiterie est responsable de leurs difficultés ; mais elles doivent toutes beaucoup à la laiterie ». Ce discours illustre la volonté du gérant de souligner les différences de stratégies qui existent entre la laiterie et les transformatrices.

De fait, bien qu'elles considèrent la minilaiterie comme un concurrent, les femmes entretenaient néanmoins de bonnes relations avec elle. Selon la transformatrice F3 : « La laiterie est notre fournisseur en lait cru pendant la saison sèche. En plus, nous avons des liens de parenté avec certains membres de la laiterie qui renforcent les relations de confiance que nous pouvons établir avec cette entreprise. Par exemple, nous avons des affinités avec le gérant de la laiterie, ce qui nous permet d'acheter le lait à crédit. C'est aussi grâce à la laiterie qu'une quantité importante du lait de vache est valorisée pendant la période de forte production, alors que les producteurs ne savent pas quoi faire de tout leur lait. C'est aussi grâce à la laiterie que les consommateurs ont du lait de bonne qualité. La laiterie a enfin permis la création d'une association de producteurs ».

Ces propos soulignent finalement la relation de complémentarité qui existait entre les transformatrices et la laiterie. Les transformatrices artisanales bénéficiaient ainsi directement de la proximité de la laiterie, notamment pour la fourniture en lait. Mais elles bénéficiaient aussi d'avantages indirects liés au «prestige » du lait de la laiterie.
La « réputation » du village de Kasséla auprès des consommateurs de Bamako leur permettait en effet de vendre leur lait à un prix élevé, le même que celui du lait produit par la laiterie.

\section{Analyse financière}

Les coûts de transformation du lait cru variaient d'une femme à l'autre. La plus grande part des dépenses concernait les services (73 p. 100 pour F1, 78 p. 100 pour F2 et 81 p. 100 pour F3), c'est-à-dire les frais de transport jusqu'à Bamako et le bois pour le chauffage du lait. Les femmes payaient 500 Fcfa par jour au poste de contrôle douanier de Niamana (poste d'entrée ou de sortie de la ville de Bamako) soit 15000 Fcfa par mois pour chacune. Au total, et avant rémunération du travail familial, les coûts de transformation du litre de lait étaient faibles : 19 Fcfa le litre en moyenne contre 30 à $34 \mathrm{Fcfa}$ à la laiterie. Il faut noter que c'est la transformatrice qui n'utilisait pas de poudre de lait qui obtenait les marges les plus élevées (tableau IV).

L'activité était rentable pour les trois transformatrices. Le rapport bénéfice sur coûts était en moyenne pour toutes les trois de 25 et 20 p. 100, respectivement pour les mois de juin et juillet (tableau V) tandis que la laiterie présentait un rapport de 3 et 3,4 p. 100, respectivement pour le mois de juin et juillet de la même année (2006) (tableau VI). Cependant, l'activité était très sensiblement plus rentable chez la femme F3 (tableau V). Cette différence s'explique probablement par la plus grande maîtrise des procédés de transformation par cette transformatrice, par l'importance de ses ventes au détail et par la plus grande diversité des produits qu'elle commercialisait sur le marché. Les bénéfices mensuels nets des transformatrices étaient élevés. En effet, avec environ le même volume de lait que celui traité par la laiterie, ces trois femmes arrivaient à dégager des marges bénéficiaires plus importantes (tableaux $\mathrm{V}$ et $\mathrm{VI}$ ).

\section{Stratégies d'adaptation des femmes}

Comme relevé plus haut, les transformatrices de Kasséla proposaient aux consommateurs une gamme variée de produits. Elles s'étaient en particulier spécialisées dans la production du ghee qui non seulement peut être conservé pendant longtemps sans réfrigération, mais qui répond aussi à un marché urbain sans cesse croissant. De plus, elles permettaient aux populations du village d'accéder régulièrement à du lait cru utilisé notamment pour des usages religieux. Dans cette région, en effet, de nombreux sacrifices se faisaient avec du lait cru.

Pour assurer l'écoulement régulier de leurs produits, ces femmes fidélisaient leur clientèle en s'appuyant sur un réseau de clients très élaboré, basé sur des relations personnelles. Pour les consommateurs réguliers, les femmes fournissait parfois du lait à crédit.

\section{Tableau IV}

Variation des coûts de transformation du lait cru et des marges bénéficiaires (en Fcfa) chez les femmes en juin et juillet 2006

Juin 2006

Femme Marge brute/L Coût de production/L Marge nette/L
Juillet 2006

Marge brute/L Coût de production/L Marge nette/L

$\begin{array}{lrrrrrr}\text { F1 } & 80 & 28 & 52 & 73 & 24 & 49 \\ \text { F2 } & 75 & 16 & 59 & 57 & 14 & 44 \\ \text { F3 } & 92 & 13 & 74 & 139 & 18 & 120 \\ \text { Moyenne } & 82 & 19 & 62 & 90 & 19 & 71\end{array}$


Tableau V

Résultats obtenus par les trois femmes en juin et juillet 2006

\begin{tabular}{llccccc} 
Femme & Mois & $\begin{array}{c}\text { Vol. de lait } \\
\text { traité (L) }\end{array}$ & $\begin{array}{c}\text { Charges } \\
\text { (Fcfa) }\end{array}$ & $\begin{array}{c}\text { Recettes } \\
\text { (Fcfa) }\end{array}$ & $\begin{array}{c}\text { Marges brutes } \\
\text { (Fcfa) }\end{array}$ & $\begin{array}{c}\text { Rapport Bénéfice/ } \\
\text { coût (\%) }\end{array}$ \\
\hline F1 & Juin & 4650 & 885810 & 1074990 & 189210 & 21 \\
& Juillet & 4030 & 952010 & 1164990 & 243690 & 26 \\
F2 & Juin & 17730 & 3831060 & 4743750 & 912690 & 24 \\
& Juillet & 22041 & 4548537 & 4689510 & 287700 & 7 \\
F3 & Juin & 15330 & 3699810 & 4666500 & 966690 & 26 \\
& Juillet & 16136 & 3888609 & 5134500 & 1371330 & 36 \\
Total & Juin & 37710 & 8416680 & 10485240 & 2068590 & 25 \\
& Juillet & 42207 & 9389156 & 10989000 & 1902720 & 20
\end{tabular}

\section{Tableau VI}

Résultats obtenus à la laiterie de Kasséla en juin et juillet 2006

\begin{tabular}{lccrcc} 
Mois & Vol. de lait traité (L) & Charges (Fcfa) & Recettes (Fcfa) & Marges brutes (Fcfa) & Rapport bénéfice/coût (\%) \\
\hline Juin & 27894 & 7847350 & 8083250 & 235900 & 3 \\
Juillet & 37804 & 10918168 & 11286700 & 368531 & 3,4
\end{tabular}

Ces réseaux de clientèles leur garantissaient un écoulement régulier de leur lait, même pendant des périodes d'abondance de lait où les risques de mévente étaient importants (hivernage). Pour augmenter les quantités de lait transformé, les femmes F1 et F2 incorporaient du lait en poudre. Cette pratique leur permettait d'éliminer « l'effet saison » et de maintenir pendant la saison sèche un volume d'activité et de revenu régulier.

Des discussions avec les femmes de Kasséla, il est ressorti un besoin urgent d'améliorer leurs conditions de travail (transport de leurs produits vers les pôles de consommation) et de mettre en place une organisation professionnelle collective. Cela passe par un regroupement souhaitable entre les transformatrices de lait à Kasséla, d'une part, pour une meilleure organisation de l'activité en concertation avec la laiterie afin de maintenir la complémentarité et de parvenir à améliorer la qualité, d'autre part, pour un accès aux financements permettant l'acquisition d'un moyen de transport autonome.

Ces femmes ne manquaient pas d'initiative pour la résolution de leurs problèmes quotidiens. Elles ont plusieurs fois sollicité des microcrédits auprès des institutions de microfinance, mais leurs projets avaient peu de chances d'être financés, en raison du caractère jugé très risqué de leur activité. Le regroupement souhaité des femmes leur aurait conféré plus de crédibilité pour accéder au microcrédit en proposant une garantie collective. Le soutien des initiatives de ces femmes était possible mais restait conditionné à un engagement de leur part pour améliorer la qualité des produits qu'elles mettaient en vente sur le marché.

Au final, doit-on considérer l'initiative des femmes de Kasséla comme une initiative limitée en nombre (trois entreprises individuelles) et dans l'espace (périphérie urbaine de Bamako) ? S'agit-il de stratégies isolées ou au contraire d'un témoignage d'une dynamique d'ensemble de la filière laitière autour des villes africaines ?
DISCUSSION : PLACE ACTUELLE DE LA FEMME DANS LA FILIERE LAITIERE EN AFRIQUE DE L'OUEST

\section{Tradition et changements de la division sexuelle des tâches laitières}

Dans la plupart des sociétés pastorales africaines, la gestion du lait est une activité traditionnellement dévolue à la femme. Au sein du troupeau familial, il revenait à l'épouse ou aux filles de gérer les opérations telles que la traite, la distribution du lait pour la consommation au sein du ménage, la transformation, le troc (en général contre les céréales) et la vente du lait (16). Dans la région de Kolda (au sud du Sénégal), par exemple, les tâches traditionnellement dévolues aux femmes concernaient la traite, la transformation du lait en lait caillé et huile de beurre, et la commercialisation des produits. A l'homme revenait le gardiennage, la conduite au pâturage, l'abreuvement, les soins aux animaux, le transport et l'échange des animaux sur pied (1). Les hommes tiraient ainsi traditionnellement profit de la vente d'animaux du troupeau et les femmes du commerce du lait (8).

Cependant, aujourd'hui, au sein des sociétés pastorales, la répartition sexuelle des tâches apparaît beaucoup plus complexe qu'elle n'y paraît, et en forte mutation. La traite est parfois le domaine réservé des femmes ; c'est le cas chez les Arabes du centre du Tchad (12), ou dans le Ferlo au Sénégal. Dans d'autres contextes, les hommes et les femmes participent conjointement à ce travail ; c'est le cas chez certains groupes peuls d'Afrique de l'Ouest (1, 10, 16). Dans certains campements, les «droits » sur le lait et les produits laitiers font l'objet d'une redistribution au sein des ménages. Dans le delta du fleuve Sénégal, par exemple, une part significative du lait produit échappe au «contrôle » des femmes peules 
et même aux femmes en général (7). Ces mutations sont dues aux transformations des systèmes de production et surtout à la mise en place de systèmes de collecte gérés par les hommes. Plusieurs études soulignent ainsi l'implication de plus en plus importante des hommes dans la transformation (unités de pasteurisation) et dans la commercialisation du lait (vente directe par les producteurs) (1). Par ailleurs, lorsque le lait est collecté tous les matins pour être acheminé à une laiterie, c'est l'homme qui est en général mobilisé et qui reçoit les revenus de la collecte $(8,10,11)$.

Pourtant, malgré cette perte de contrôle, les femmes restent finalement présentes dans l'activité laitière. La comparaison de trois régions laitières (Kasséla au Mali, St Louis au Sénégal et Niamey au Niger) permet de mettre en évidence trois cas de figures relatives à l'évolution du rôle des femmes dans les systèmes laitiers : la perte de prérogatives laitières, la survivance d'activités féminines traditionnelles et la participation aux nouvelles activités économiques de la filière.

\section{Perte de prérogatives des femmes}

Dans les cas où les femmes sont complètement exclues des nouveaux circuits du lait, on peut dire qu'elles perdent leurs prérogatives. A St Louis comme dans toutes les grandes villes du Sénégal, les grossistes et les boutiquiers sont essentiellement des hommes; les femmes sont exclues du commerce et même de la transformation de la poudre de lait en lait caillé. Tout le secteur formel leur échappe (9). Dans ce cas, on peut parler de déféminisation de la filière. Dans de telles configurations, les femmes deviennent économiquement vulnérables si elles ne parviennent pas à réorienter leurs activités vers de nouvelles sources de revenus.

\section{Survivance des activités féminines traditionnelles}

Dans certains cas, on observe plutôt une survivance des activités laitières traditionnelles. Une bonne illustration en est donnée par l'exemple des femmes de Kasséla qui, par des stratégies personnelles, sont parvenues à maintenir leur activité depuis environ 25 ans, malgré les transformations radicales du système laitier local. Leur résilience économique (c'est-à-dire leur capacité à conserver des parts de marché) s'explique par leur capacité à proposer des produits différents de ceux de la laiterie. Dans ce cas, les femmes parviennent à dégager des profits substantiels.

Ainsi, les produits vendus par les minilaiteries ne sont pas toujours concurrents des produits fermiers vendus traditionnellement par les femmes $(10,13)$. A Kasséla, par exemple, la laiterie propose deux types de produits (féné et lait pasteurisé) alors que les femmes vendent cinq types de produits (lait cru, lait chauffé, féné, lait caillé et ghee). De manière similaire, on observe que dans de nombreux cas, la répartition sexuelle des tâches fait l'objet d'une concertation ou même d'une négociation entre hommes et femmes. Par exemple, lorsque les centres de consommation sont proches des zones de production, le lait cru ou transformé est vendu directement par les femmes et les recettes sont gérées par celles-ci. C'est le cas dans le village de Kornaka (au centre du Niger) où l'on peut voir le jour de marché une trentaine de femmes peules vendre le lait, le beurre ou le fromage (16), mais lorsque les points de vente sont très éloignés des zones de production, les hommes se chargent de la vente des produits laitiers (beurre et généralement fromages).

La gestion des recettes générées par ces ventes peut alors faire l'objet d'une discussion au sein du ménage. Les innovations ne sont donc pas toujours synonymes de mise à l'écart des femmes car, parfois, le revenu continue d'être géré en concertation entre l'homme et la femme. Il peut ainsi arriver que l'homme reverse à son épouse une partie du revenu mensuel généré par la livraison du lait à une laiterie. Souvent, aussi, c'est seulement une partie de la production du troupeau qui est vendue à la laiterie, une autre partie peut être transformée et vendue par l'épouse qui conserve alors ses activités de commerce laitier (10).

\section{Participation des femmes aux nouveaux métiers de la filière}

Enfin, les nouveaux métiers du lait, associés à l'émergence des minilaiteries (collecte à vélo, points de vente, etc.) n'éliminent pas complètement les femmes du système. D'une part, les « anciens » métiers traditionnellement assurés par les femmes, comme le commerce du lait caillé ou de l'huile de beurre, les livraisons de lait au porte-à-porte, etc., ne disparaissent pas complètement On assiste plutôt à une diversification des métiers du lait plutôt qu'au remplacement de certains métiers par d'autres. D'autre part, il s'avère que les femmes se sont aussi investies dans les nouveaux métiers du lait : elles sont ainsi de plus en plus présentes dans le fonctionnement et la gestion des minilaiteries.

Un nombre non négligeable de minilaiteries gérées par des groupements de femmes ont été mises en place dans plusieurs zones, comme par exemple dans la vallée du fleuve Sénégal. Ces petites entreprises exploitent en moyenne 60 litres de lait par jour sur un rayon d'approvisionnement de $15 \mathrm{~km}$ (2). En développant ces activités, ces femmes gèrent des entreprises dont elles sont les propriétaires ou les gérantes. Dans les systèmes de collecte organisée, les femmes continuent aussi de participer aux activités de transformation du lait par l'intermédiaire des emplois au sein des laiteries. Dans les minilaiteries du Mali et du Sénégal, le personnel est presque exclusivement féminin et en général d'origine peule $(8,15)$.

\section{Participation des femmes et projets de développement}

Les projets de développement de la filière laitière en cours doivent s'inspirer des expériences passées pour limiter les risques de marginalisation des femmes liés à la mise en place de minilaiteries. Déjà au Mali, les projets laitiers en cours de réalisation reconnaissent la nécessité d'impliquer tous les acteurs de la filière à travers des démarches de concertation et de mise en relation, afin d'éviter les mécanismes d'exclusion sociale (4). Cette implication concerne prioritairement les femmes et les bergers qui sont les catégories socioprofessionnelles qui risquent le plus d'être affectées par la modernisation et l'industrialisation de la filière laitière. A Cinzana (zone périurbaine de Ségou au Mali), par exemple, l'accent est mis sur une participation active et massive des femmes à toutes les étapes de la mise en œuvre des futurs projets laitiers $(4,7)$. Les discussions avec les acteurs montrent clairement que la concertation et la négociation s'imposent comme des pré-conditions pour la mise en place du projet, et une gestion partagée et équitable des revenus du lait entre les hommes et les femmes (14).

\section{CONCLUSION}

L'analyse des transformations des systèmes laitiers montre que la mise en place des minilaiteries rurales a fortement affecté les activités laitières des femmes. Cependant, les femmes ont su résister à ces recompositions. Elles sont restées très présentes dans la transformation artisanale, même si peu de travaux avaient jusqu'à présent été menés pour étudier ces micro-entreprises individuelles. L'analyse des activités des transformatrices de Kasséla montre que ce type d'entreprise est rentable, et que ces transformatrices proposent des produits relativement spécifiques qui ne sont pas commercialisés par les minilaiteries. 
Cependant, ces activités féminines de transformation laitière ont gardé en partie un caractère très informel ; ces micro-entreprises gagneraient à se structurer collectivement. Les transformatrices de Kasséla ont besoin, par exemple, pour améliorer la qualité de leurs produits, d'une organisation qui leur permettrait de bénéficier de formations techniques et de crédits afin de résoudre leur problème de transport.

Les décideurs et les acteurs de développement devront à l'avenir impliquer plus implicitement les femmes dans les processus d'initiation des projets laitiers. Dans le but de promouvoir l'équité sociale, il est important de bien maîtriser les déterminants des changements et de tenir compte des fonctions et des diverses activités du genre à tous les niveaux de la filière. Une approche qui favoriserait l'intégration de toutes les catégories sociales (femmes, bergers, propriétaires) dans les instances de décisions et l'accès aux différents services de production contribuerait à assurer aux populations des moyens de subsistance durables.

\section{Remerciements}

Cette étude a été conduite dans le cadre du thème transversal sur le pastoralisme «Extensive Production System » du Pôle de partenariat de recherche Nord-Sud (Nccr-North-South), partenariat pour l'atténuation des syndromes du changement global. Le Nccr-NorthSouth est cofinancé par la Direction pour le développement de la coopération suisse (DDC) et le Fond national suisse de recherche scientifique (FNS). Nous remercions VSF-Suisse pour l'appui à l'accès aux informations et Mme Maty Ba Diao (Insah) pour ses commentaires sur le texte et ses précieux conseils.

\section{BIBLIOGRAPHIE}

1. BA DIAO M., SENGHOR C.D., DIAO B., 2002. Les femmes dans la filière lait périurbaine au Sénégal. Cas de la région de Kolda. Revue Elev. Méd. vét. Pays trop., 55 : 299-304.

2. BA DIAO M., TRAORE E.H., DIENG A., SALL C., SOW O.S., TONFIO R., 2004. Petites entreprises de transformation et développement laitier dans la vallée du fleuve Sénégal. RASPA, $1: 25-30$.

3. BEAGGES, 2005. Plan d'aménagement et de gestion du domaine d'intervention forestier de Kasséla (forêt classée de la Faya). Bamako, Mali, ministère de l'Environnement, $40 \mathrm{p}$

4. BONFOH B., DICKO M.D., DEMBA D., KOUYATE H., FANE A. FOKOU G., 2007. Extension du modèle de développement laitier en milieu rural : commune rurale de Cinzana (région de Ségou). Complexe laitier comme réponse à un besoin des agro-pasteurs. Etude de faisabilité Bamako, Mali, Institut du Sahel / Nccr North-South Bamako, 33 p.

5. BONFOH B., FANE A., NETOYO L., MBAYE Y., SIMBE C.F., ALFAROUKH I.O., NICOLET J., FARAH Z., ZINSSTAG J., 2004. Collecte et distribution du lait produit localement en zone urbaine de Bamako (Mali). Revue Etud. Rech. Sahél., 8-9 : 13-18.

6. BONFOH B, FANE A., STEINMANN P, HETZEL M, TRAORE A.N, TRAORE M., SIMBE C.F., ALFAROUKH I.O., NICOLET J., AKAKPO J.A., FARAH Z., ZINSSTAG J., 2003. Qualité microbiologiques du lait et des produits laitiers vendus au Mali et leurs implications en santé publique. Revue Etud. Rech. Sahél., 8-9 : 19-27.

7. BOUTINOT L., 2006. Le beurre et l'argent du beurre : intérêt et limites du concept " genre » dans les études préalables aux projets de développement. Bull. APAD (20). http://apad.revues.org/document251.html

8. CORNIAUX C., 2005. Gestion technique et gestion sociale de la production laitière : les champs du possible pour une commercialisation durable du lait. Cas des modes de production actuels du delta du fleuve Sénégal. Thèse Doct., INA, Paris-Grignon, France, 258 p.

9. DIA D., 2004. Une tradition sociale bousculée par l'avènement des mini laiteries. Http://www.laitsain.com/forum/index.php?fid=4 (consulté 21 avr. 2007)
10. DUTEURTRE G., 2004. Genre et lait. Http://www.laitsain.com/forum/ index.php?fid=4 (consulté 21 avr. 2007)

11. FAO, 2004. Lait et produits laitiers. Questions de parité hommesfemmes dans le secteur laitier. Rome, Italie, FAO. Fao.org

12. MIAN O.K., 2004. Le nouveau rôle des femmes dans le commerce du lait frais à N'Djamena. http://www.laitsain.com/forum/index.php?fid=4 (consulté 21 avr. 2007)

13. SALL A., 2004. Complémentarité ou concurrence dans les systèmes de collecte de lait ? http://www.laitsain.com/forum/index.php?fid=4 (consulté 21 avr. 2007)

14. SCHNEIDER M., KOUYATE H., FOKOU G., BONFOH B., 2007. Microfinance dans les systèmes de production animale au Sahel. Bamako, Mali, Institut du Sahel / Nccr North South,15 p.

15. SERY A., 2006. Impacts des minilaiteries sur les transformations socioéconomiques et culturelles au Mali. Rapport d'évaluation. Berne, Suisse, VSF.

16. VIAS G.F.S., 2004. La co-gestion du lait dans les sociétés pastorales en pleine mutation au Niger. http://www.laitsain.com/forum/index. php?fid=4 (consulté 21 avr. 2007)

17. VSF Suisse, 2006-2007. Rapport. Berne, Suisse, VSF.

18. WANE A., 2006. Economie du pastoralisme : une analyse bibliographique. Afrique de l'Ouest. Dakar, Sénégal, Wisp/lucn.

19. WULLSCHLEGER S., EGGER S., LACROIX C., BONFOH B., TEMBELY S., FARAH Z., MEILE L., 2009. Antibiotic resistance analysis of Enterococcus sp. isolates from the fermentation process of féné, a spontaneously fermented sour-milk product in Mali. In: Wullschleger S., Biodiversity and microbial safety of artisanal Malian sour milk féné and development of adapted starter cultures for controlled production. Zurich, Germany, ETH, p. 72-86

Accepté le 01.02.2010 


\section{Summary}

Schneider M., Kouyaté H., Fokou G., Zinsstag J., Traoré A., Amadou M., Bonfoh B. Dynamics of Women's Adaptation to Change in a Suburban Dairy System in West Africa

In West Africa, through dairy development projects, new dairy models emerge alongside the traditional production system as a result of technology innovations and dairy policy. However, the creation of milk collection centers, small dairy farms and dairy plants disrupts the traditional social organization of the dairy system and contributes to gender marginalization. Some women are dispossessed of the activities of milk collection, marketing, and management of milk revenue to the profit of men herders or owners. This process may thus contribute to the loss of their financial autonomy. To understand better these changes, a case study of three milk processing women practicing their trade around Kassela small dairy plant, $40 \mathrm{~km}$ from Bamako, was conducted in Mali. Questionnaires and focus group interviews were used to assess the women's adaptation level to the dynamics of change in the local dairy subsector. In response to the creation of a small dairy farm managed by men, they initiated mechanisms of resilience by adding imported dry milk, diversifying the product offer on the markets, and developing consumers' loyalty. These strategies enabled them to secure their livelihoods in spite of their loss of market shares to the dairy plants. Using local techniques, each woman processed on average 468 liters of milk per day representing one third of the dairy plant production with a ratio benefit/cost greater than $20 \%$. Today, dairy development projects take more and more into account the consequences brought about by innovation.

Keywords: Milk product - Milk collection - Modernization Role of women - Social change - West Africa.

\section{Sumario}

Schneider M., Kouyaté H., Fokou G., Zinsstag J., Traoré A., Amadou M., Bonfoh B. Dinámicas de adaptación de las mujeres frente a las transformaciones de los sistemas lecheros peri urbanos en Africa del Oeste

En Africa del Oeste, los nuevos modelos de recolección de leche, por unidades de transformación, son el reflejo de innovaciones tecnológicas e institucionales promovidas por las políticas y los proyectos de desarrollo de la filial. Sin embargo, la creación de centros de recolección, de mini lecherías o de industrias lecheras trastorna la organización social tradicional del sistema lechero y contribuye a una "desfeminización" de la filial. Ciertas mujeres se encuentra desposeídas de la actividad de recolección - comercialización y de la gestión de los ingresos de la leche a favor de los pastores o propietarios hombres. Este proceso es por lo tanto susceptible de contribuir a la pérdida de la autonomía financiera de éstas. Un estudio de casos Ilevado a cabo en Malí, con tres mujeres transformadoras, ejerciendo alrededor de la mini lechería de Kassela, a 40 kilómetros de Bamako, permitió aclarar estas evoluciones. A partir de cuestionarios y de entrevistas semi estructurados, el estudio permitió medir el grado de adaptación de las mujeres en esta dinámica de transformación de la filial lechera local. Frente al surgimiento de una mini lechería, gestionada por hombres, ellas iniciaron varios mecanismos de resiliencia. Esto incluyó desde la incorporación del polvo de leche importado y la diversificación de los productos que ellas llevaban al mercado, hasta la mantener la lealtad de la clientela. Estas estrategias les permitieron asegurar sus medios de subsistencia, a pesar de la acaparación de las lecherías de una parte del mercado. Con estas técnicas artesanales, cada una de ellas transformaba un promedio 468 litros de leche por día, o sea un tercio de la producción total de la lechería de Kassela, con una relación ganancia sobre costo superior a 20 \%. Hoy, los proyectos de desarrollo lechero tienden cada vez más a discutir las implicaciones sociales de las innovaciones propuestas.

Palabras clave: Producto lácteo - Recogida de leche Modernización - Papel de la mujer - Cambio social - Africa occidental. 